looks like for them individually. The project aims to utilise a collaborative, interdisciplinary, and whole team approach.

Approach Used The project has taken a multi-faceted approach:

Leadership

- Appoint a project lead

- Set up a multi-professional task and finish group

- Champion and role model a different way of thinking.

Training

- Delivery of training sessions from ward to Board

- Develop core enablement and rehabilitation competencies for assistant staff

- Incorporate rehabilitation and enablement approach into staff and volunteer induction.

Service development

- Implementation of a programme of circuit exercise in the gym

- Development of 'What Matters to Me' documentation

- Implementation of a self-management 'Positive Steps to Well-being' programme

- Development of a visual manual handling plan for patients on the Inpatient Unit

- Pilot mirroring package of care on the Inpatient Unit prior to discharge home

- Development of a new volunteer community enablement assistant role.

Organisational structure and process

- Staff job descriptions/volunteer role profiles to reflect rehabilitation and enablement

- Incorporate reflection and evidence of rehabilitative palliative care practice in the appraisal process

- New healthcare assistants shadow the Allied Health Professions team to learn rehabilitative skills in practice.

Outcomes On completion of the project, the hospice aims to increase our total rehabilitative palliative care score to over 80/111 from 29/111 in August 2015 and 51/111 in August 2016 (Tiberini \& Richardson, 2015).

Conclusion Effective implementation of an enabling approach has required a stepped approach, an understanding of organisational readiness, and key cultural barriers.

\section{P-144 WHOSE CHOICE IS IT ANYWAY?}

Maureen Howe, Anthony Walsh, Jackie Green, Margaret Hollinshead. St Catherine's Hospice, Lancashire, UK

\subsection{6/bmjspcare-2017-hospice. 169}

Background The rehabilitation team consists of two part-time physiotherapists, totalling just over one whole-time equivalent. Most of the time they work single-handedly. Whilst reviewing their roles, the Rehabilitation Team identified two main concerns.

When patients want to do an activity that requires more than one person to facilitate it, the physiotherapist would have to wait for another member of staff to be available.

There were missed opportunities for some patients for example; those that were independent and in the hospice for symptom control.

Aims To increase the rehabilitation resource.

Every patient to have options for activities, and enable them to continue these outside of the hospice environment.
Method

- Patient focus groups met over a three month period to determine which patient needs were not being met

- Applied to St James's Place Foundation for funding of $£ 35000$ to establish a training programme for volunteers

- Recruited and trained a team of volunteers, two people per week day.

Results Nine volunteers trained - induction, full moving and handling, some had fatigue, anxiety and breathlessness management training. Each volunteer has an average of 5 contacts per day. The rehabilitation team now includes trained volunteers. They support the nursing and rehabilitation teams to give patients an increased choice of activities and provide another pair of hands for mobility or functional assessments.

'Jonny was really good with my Mum this morning, showing her how to get out of bed and spending time with her in Day Therapy' - Daughter of the patient

'Having nursing assistants with enhanced skills and knowledge working alongside volunteers has had such a positive impact on the team's ability to carry out the necessary assessments to meet our patients' needs without delay.' - Sister InPatient Unit

Future Developments

- Consideration of a seven day service

- Further training of the volunteer team.

\section{P-145 LIVING WELL WITH HEART FAILURE: A CREATIVE PARTNERSHIP APPROACH}

Sian Cole. Longfield, Gloucestershire, UK

10.1136/bmjspcare-2017-hospice. 170

Background One in 15 people aged $75-84$ in the UK have some degree of heart failure. Longfield working collaboratively with the Heart Failure Clinical Nurse Specialist team developed and piloted a six-week rehabilitative heart failure programme.

Aims To develop, pilot and evaluate a heart failure programme. The programme used a combined clinical and creative approach, bringing together the skills of Heart Failure CNS and Creative Therapists. The objectives of the programme were to increase exercise tolerance, knowledge and understanding of heart failure, improve feelings of well-being and support patients to achieve better health outcomes, such as decrease in medical interventions.

Methods The six-week programme had three main components: clinical, creative therapy and exercise in the form of Tai Chi.

Topics covered during the programme included managing breathlessness and fatigue, advance care planning, nutrition, weight loss, medication, mood and stress management. Sessions concluded with an exercise component that consisted of Tai Chi.

Results Two programmes were delivered with 13 attendees in total. In addition we ran a taster/introductory session to which carers and participants were invited, to give them an overview of the programme. Qualitative and quantitative patient feedback was gathered at the beginning and at the end of both programmes. Feedback was positive with respondents feeling more able to engage in exercise and increased confidence in managing symptoms and medication. In the second pilot 